\title{
Productividad del aserrado de machiche (Lonchocarpus castilloi Standl), en Tres Garantías, Quintana Roo, México
}

\author{
Productivity of sawing the machiche wood \\ (Lonchocarpus castilloi Standl), \\ in Tres Garantías, Quintana Roo, Mexico
}

\author{
Alejandra Acosta-Ramos ${ }^{1}$ y Joel Martínez-López 1
}

\begin{abstract}
RESUMEN
Se determinaron rendimientos y tiempos de aserrado, con el propósito de conocer la productividad del aserrío de Lonchocarpus castilloi Standl. (machiche). El rendimiento se determinó al relacionar el volumen de madera en rollo y el volumen de madera aserrada de 815 trozas. Se determinaron tiempos de asierre, empleando el método del tiempo total en la sierra principal. En $80 \%$ de las trozas se registraron diámetros de $30 \mathrm{~cm}$ a $40 \mathrm{~cm}$ y en $94 \%$, longitudes de $2 \mathrm{~m}$ a $3 \mathrm{~m}$. El volumen total fue de 194,6 $\mathrm{m}^{3}$ rollo fuste sin corteza ( $\mathrm{rfsc}$ ), que al procesarse generaron un volumen de $80,8 \mathrm{~m}^{3}$ aserrados. Esto indica que para producir un metro cúbico de madera aserrada se requieren $2,45 \mathrm{~m}^{3}$ rfsc de machiche. El coeficiente de aserrío real global fue $41,3 \%$; el coeficiente por clases de tablas fue $43,1 \%$ en tablas de albura, $34,1 \%$ en tablas de duramen y $22,8 \%$ en tablas de rechazo. El tiempo trabajado productivo fue de $68 \%$, el tiempo trabajado no productivo fue de $19 \%$ y el tiempo no trabajado justificado fue de $13 \%$. La productividad se ubicó en $0,57 \mathrm{~m}^{3} /$ hora. Los indicadores de productividad determinados proveen información útil para la administración eficiente del aserradero ejidal.
\end{abstract}

PALABRAS CLAVE:

Coeficiente de rendimiento, maderas duras tropicales, proceso de aserrado, tiempos de aserrado.

\begin{abstract}
Yields and sawing time were determined in order to know the sawmilling productivity of Lonchocarpus castilloi Standl. (machiche). The yields were determined by relating the volume of logs and sawn timber volume of 815 logs. Milling times were determined using the total time method in the main saw. Eighty percent of logs recorded diameters from $30 \mathrm{~cm}$ to $40 \mathrm{~cm}$, and $94 \%$ of them had lengths from $2 \mathrm{~m}$ to $3 \mathrm{~m}$. The total volume processed was $194,6 \mathrm{~m}^{3}$ round wood without-bark (rwwb), that generated a volume of $80,8 \mathrm{~m}^{3}$ of sawn lumber. This indicates that to produce one cubic meter of lumber, $2,45 \mathrm{~m}^{3}$ of machiche are required. The actual global sawmill coefficient was $41,3 \%$, the coefficient for grades of boards was $43,1 \%$ in boards of sapwood, $34,1 \%$ in boards of heartwood, and $22,8 \%$ in boards with rejection grade. The productive time was $68 \%$, the non-productive time $19 \%$, and the leisure time $13 \%$. The productivity was at $0,57 \mathrm{~m}^{3 /}$ hour. The productivity indicators identified, provide useful information for the efficient administration for sawmill of the ejido.
\end{abstract}

KEY WORDS:

Yield coefficients, tropical hardwoods, sawing process, sawing times. 


\section{INTRODUCCIÓN}

La industria del aserrío es considerada la segunda más importante dentro del sector forestal en México, tanto por el volumen que procesa como por el número de plantas que existen. Se caracteriza por ser poco intensiva en capital y con instalaciones de baja productividad (FloresVelázquez et al., 2007; Fuentes-López et al., 2006). En las regiones de bosque tropical se encuentra un menor número de plantas industriales, aunado a un mayor rezago industrial. Esto por lo general se refleja en mayores dificultades en el aprovechamiento e industrialización de especies tropicales (Flores-Velázquez et al., 2007).

No obstante, a nivel nacional Quintana Roo es el segundo estado productor de maderas tropicales duras aserradas, superado sólo por Campeche. Las principales maderas duras comercializadas son Metopium brownei (Jacq.) Urban, Lonchocarpus castilloi (Standl), Lisiloma bahamensis (Benth) y Caesalpinia platiloba (L). En menor cantidad se procesan, Bucida buceras (L), Swarzia cubensis (Britt. \& Wilson) Standl. y Sickingia salvadorensis (Standl.) Steyerm (Forster et al., 2002).

Ante el rezago industrial en regiones tropicales es prioritario que la industria considere elementos básicos para lograr la eficiencia de transformación de los recursos maderables. Diversos autores, como Biasi y Rocha (2006), señalan que toda industria maderera que no se ocupe en mejorar sus rendimientos, asume el riesgo de perder competitividad y paralizar sus actividades. Estudios como la determinación del coeficiente de rendimiento y la determinación de tiempos y movimientos, son indicadores básicos para evaluar la eficiencia en aserraderos (Zavala y Hernández, 2000; Quirós et al., 2005; Nájera-Luna et al., 2011).
La eficiencia del aserrado se calcula a partir de un cociente entre la estimación del volumen de trozas que ingresan y el producto bruto de la madera aserrada que egresa. Este porcentaje se utiliza para la planificación y análisis económico de una empresa (Martínez-Pastur et al., 2002; Aguilera et al., 2005; Quirós et al., 2005; Valerio et al., 2007). Los tiempos y movimientos son una técnica de medición del trabajo empleado para registrar los tiempos y ritmos de trabajo de una tarea definida (OIT, citado por Ramírez, 1995). Conocer cómo se utiliza el tiempo, permite mejorar las condiciones de trabajo, optimizar los costos de operación y facilitar el control de la producción durante la ejecución (Chávez y Guillen, 1997).

Diversos estudios se han enfocado en el análisis del rendimiento y la productividad en especies de bosque templado, especialmente para coníferas y encinos (Juacida y Saldivar, 2000; Meneses y Guzmán, 2000; Zavala y Hernández, 2000; García et al., 2001; Zavala, 2003; Murara et al., 2005; Nájera et al., 2006; Velázquez et al., 2006; Valerio et al., 2007; García et al., 2009; Estévez et al., 2010 y Nájera-Luna et al., 2011). Para maderas tropicales se han realizado estudios enfocados al análisis del proceso de asierre y a la determinación de rendimientos (Moya y Córdoba, 1995; Guillén y Chávez, 1996; García y Moya, 1998; Quirós et al., 2005; Biasi y Rocha, 2006; Rueda-Sánchez et al., 2007 y Valera y Salvador, 2009). Las publicaciones de estudios de tiempos y movimientos del aserrado son escasas para este grupo de especies.

\section{OBJETIVOS}

Aportar información sobre la productividad del aserrío de maderas duras tropicales en Quintana Roo, por medio de un estudio del coeficiente de rendimiento $y$ 
de un estudio de tiempos del proceso de aserrado de machiche (Lonchocarpus castilloi Standl.).

\section{METODOLOGÍA}

El presente trabajo se llevó a cabo en el ejido Tres Garantías, el cual se encuentra al sur del estado de Quintana Roo, México y pertenece al municipio de Othón Pompeyo Blanco; se ubica geográficamente entre los paralelos $18^{\circ}$ y $19^{\circ}$ $16^{\prime} 00^{\prime \prime}$ de latitud Norte y los meridianos $88^{\circ} 52^{\prime} 00^{\prime \prime}$ y $89^{\circ} 09^{\prime} 50^{\prime \prime}$ de longitud Oeste del meridiano de Greenwich. En este estudio se utilizó la cantidad total de trozas de Lonchocarpus castilloi Standl correspondientes al volumen anual cosechado de la especie. Es pertinente mencionar que la exposición de los fustes por un año en el patio del aserradero generó su deterioro por el contacto directo con el suelo, así como grietas en las caras y a lo largo de los fustes. El saneo y seccionado de los fustes se realizó como tratamiento previo al aserrado, para eliminar zonas dañadas procurando trozas rectas. Para determinar la longitud de las trozas y la longitud y anchura de las tablas se utilizó un flexómetro; se empleó forcípula y flexómetro para determinar el diámetro de las trozas y realizar anotaciones en las tablas.

Se utilizó un aserradero mecánico, con motor estacionario a diesel de 160 caballos de fuerza, con torre cinta con volantes de $127,4 \mathrm{~cm}$ (52 pulgadas) de diámetro y cinta de $20,3 \mathrm{~mm}$ (8 pulgadas) de ancho, para el aserrado de trozas; una sierra desorilladora con dos discos de 101,6 (40 pulgadas) de diámetro, para sanear y dimensionar en ancho las tablas; un péndulo con sierra circular de $70 \mathrm{~cm}$ (24 pulgadas) de diámetro, para dimensionar en longitud las tablas; y un cabrestante (winch) eléctrico para el arrime de trozas. El aserradero con capacidad instalada de $18,88 \mathrm{~m}^{3}$ (8000 pies tabla) por turno en jornadas de 8 horas, procesa trozas de maderas blandas de $6 \mathrm{~m}$ de longitud y maderas duras hasta de $5 \mathrm{~m}$ de longitud. A la maquinaria y equipo se le realizaron adecuaciones para el aserrío de maderas duras y blandas; así también, previo al inicio del aserrado se realizó mantenimiento preventivo. Para el aserrío de maderas duras se emplearon dos sierras con revestimiento de estelita, de calibre 16 , con paso de diente de $3,81 \mathrm{~cm}$ ( $1 \frac{1}{2}$ pulgadas), una de reciente adquisición y la otra de uso.

Cubicación de trozas. A cada troza numerada se le midieron los diámetros mayor y menor con corteza, así como su longitud. El volumen individual se obtuvo empleando la fórmula de Smalian, que corresponde a la siguiente expresión (Romahn et al., 1994).

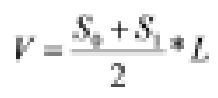

donde:

$\mathrm{V}: \operatorname{volumen}\left(\mathrm{m}^{3}\right)$

$\mathrm{L}: \quad$ longitud $(\mathrm{m})$

$\mathrm{S}_{0}$ : área de la sección menor de la troza $\left(\mathrm{m}^{2}\right)$

$\mathrm{S}_{1}$ : área de la sección mayor de la troza $\left(m^{2}\right)$

Para el aserrío de la trocería se empleó el sistema de aserrado mixto, que es el método más común y tradicional practicado en la región. Con los datos registrados de la trocería se elaboró una base de datos para su procesamiento.

Cubicación y clasificación de madera aserrada. A cada producto aserrado se le midieron sus dimensiones considerando el refuerzo, el cual es una tolerancia en 
los espesores y longitudes trabajadas por efecto del secado y el cepillado. Se empleó el flexómetro para evitar la sobrestimación o subestimación del volumen a que se incurre con el uso de la regla de cubicación. El volumen de la madera aserrada se determinó por medio de la siguiente expresión (Moya y Córdoba, 1995):

$$
V_{p}=\frac{G^{*} A^{*} L}{12}
$$

donde:

Vpt : volumen de la tabla (pies tabla)

$G$ : grueso (pulgadas)

$A$ : ancho (pulgadas)

$L: \quad$ largo (pies)

Los resultados de la cubicación se anotaron en formatos de registro denominados convencionalmente en los aserraderos tálises de producción, que llevan la cuenta de las dimensiones de los productos por día. Los productos aserrados se clasificaron con atención a dos criterios. El primero atendió a la longitud de las tablas; clasificando a las de longitud igual o menor a $1,52 \mathrm{~m} \mathrm{(5}$ pies) como tablas de cortas dimensiones y a las de longitud igual o mayor a 1,83 m (6 pies) como tablas de largas dimensiones. El segundo criterio de clasificación atendió a la calidad de las tablas, considerando la presencia de albura y duramen. Por tradición, en el estado de Quintana Roo la madera aserrada de maderas duras se clasifica como lo indica la tabla 1.

La madera con contenido de duramen ha tenido mayor precio que la madera de albura. Debido a que en el contrato de venta se especificó un mayor precio para las tablas con contenido total de duramen, en este estudio se distin- guieron los productos aserrados con contenido de albura y los productos aserrados con contenido de duramen. Por tanto, se clasificó en tablas largas de albura y duramen, tablas cortas de albura y duramen, tablas largas y cortas de rechazo. Los datos registrados en la producción de tablas se anotaron en el sistema inglés, para el procesamiento de la información, posteriormente los datos se transformaron a unidades del sistema internacional.

Determinación del coeficiente de aprovechamiento. Para obtener el nivel de transformación de la madera en rollo, se determinó el rendimiento en porcentaje, a través de la expresión utilizada por Ayerde (1993):

$$
C A=\frac{V M A}{V M R} * 100
$$

donde:

$C A$ : coeficiente de asierre

VMA: volumen de madera aserrada $\left(\mathrm{m}^{3}\right)$ VMR: volumen de madera en rollo $\left(\mathrm{m}^{3}\right)$

El coeficiente de aserrío real se estimó tomando en cuenta las dimensiones de las tablas a obtener: largas y cortas dimensiones, así como a los tipos de productos aserrados: tablas con albura, tablas con duramen y tablas tipo rechazo. La madera aserrada se estibó en una nave para secado al aire libre, donde se realizó un segundo conteo y cubicación para confirmar la información de los tálises de producción, ya que esta actividad se realizó tratando de no interferir en el proceso de producción. Aunque en el presente estudio no se planteó realizar la cuantificación de los subproductos del aserrío, su evaluación es importante porque son empleados como material para construcción rural, para elaborar 
artesanías y porque generan energía (Valerio et al., 2007). Se determinó el grado de asociación entre el volumen de madera en rollo y el volumen de madera aserrada, así como la asociación del volumen de madera en rollo y el número de piezas aserradas por día, por medio del coeficiente de correlación $r$ con base en la siguiente expresión (Gutiérrez y De la Vara, 2008):

$$
r_{\lambda \gamma}=\frac{S_{x y}}{\sqrt{S_{x x} S_{V r}}}
$$

donde:

$S_{X Y}$ : covarianza

$S_{X X}$ : varianza del volumen de madera en rollo

$S_{Y Y}$ : varianza del volumen de madera aserrada

Determinación de tiempos de asierre. En atención a los requerimientos de la administración del aserradero se determinaron tiempos productivos e improductivos del aserrado de las 815 trozas de machiche en la sierra principal. Ante esta situación fue necesario emplear el método del tiempo total, tomando datos únicamente en la estación principal de aserrado. No obstante, Bennet (1972), menciona que el análisis de una operación específica ofrece información útil para operaciones similares y además puede ser de utilidad para detectar cambios necesarios o modificaciones en el asierre de trozas; por lo anterior, el estudio de tiempos se complementó con observaciones generales en las máquinas desorilladora y despuntadora.

Para organizar el registro y análisis de la información, se adaptó la metodología definida por Chávez y Guillén (1997), que clasifica los tiempos de acuerdo con: a) tiempo trabajado, que se subdivide en trabajo productivo (asierre) y trabajo no productivo (por ejemplo: cambio de sierra), y b) yiempo no trabajado, que se subdivide en tiempo justificado (por ejemplo, falla en el sistema de energía eléctrica) y tiempo no justificado (por ejemplo, distracciones). Durante el proceso de asierre de las 815 trozas se registraron los tiempos de espera de trocería, reparaciones, mantenimiento, distracciones y reuniones. Se determinó el tiempo total empleado, que es la suma de los dos tipos de tiempos definidos. Se determinaron los principales indicadores del rendimiento, entre ellos: horas de observación, porcentaje de los tipos de tiempos empleados, número y volumen de trozas aserradas, número de trabaja-

Tabla 1. Clasificación local de maderas duras y su equivalencia con la National Hardwood Association.

\begin{tabular}{lll}
\hline Mill run Largas dimensiones & 1 & Común y mejor \\
Mill run Cortas dimensiones & 3A & Común y mejor \\
Rechazo Largas dimensiones & 2B & Común e inferior \\
Rechazo Cortas dimensiones & 3A & Común e inferior
\end{tabular}


dores, rendimiento en trozas por hora, $\mathrm{m}^{3}$ aserrados por hora, $\mathrm{m}^{3}$ aserrados por hora-hombre y productividad, calculada por medio de la ecuación 5 (García et al., 2001).

$$
P=\frac{V M A}{T P}
$$

donde:

$P$ : Productividad

VMA: Volumen de la madera aserrada $\left(\mathrm{m}^{3}\right)$

TP: Tiempo de proceso (horas)

La información obtenida, tanto para la determinación del coeficiente de aserrío, como para la determinación del tiempo trabajado y el tiempo no trabajado, se organizó en hojas de cálculo para su procesamiento estadístico.

\section{RESULTADOS Y DISCUSIÓN}

Naturaleza de la trocería. De acuerdo con las frecuencias absolutas de las categorías diamétricas de las 815 trozas que se muestran en la figura 1 , se observa que la categoría diamétrica más frecuente fue la de 35 centímetros y la menos frecuente fue la de 60 centímetros. Las medidas de dispersión obtenidas para las categorías diamétricas fueron: desviación estándar de 6,09 centímetros, coeficiente de variación de $16,71 \%$, error estándar de la media de 0,21 centímetros, coeficiente de curtosis de $0,29 \mathrm{y}$ coeficiente de asimetría igual a 0,64. De acuerdo con lo anterior, los indicadores para este conjunto de datos son altamente confiables desde el punto de vista estadístico.

Las frecuencias absolutas de las categorías de longitud de las 815 trozas se muestran en la figura 2. La categoría en longitud mas frecuente correspondió a $2,0 \mathrm{~m}$ y la categoría en longitud menos frecuente fue de $1,5 \mathrm{~m}$, mientras que el rango promedio se ubicó en la categoría de longitud de $3 \mathrm{~m}$. Sus indicadores estadísticos fueron desviación estándar de 0,45 metros, coeficiente de variación de $18,46 \%$, error estándar de la media de 0,02 metros, coeficiente de asimetría de 0,50 y coeficiente de curtosis de $-0,14$. La notable falta de estandarización en dimensiones de longitud, se debe a que

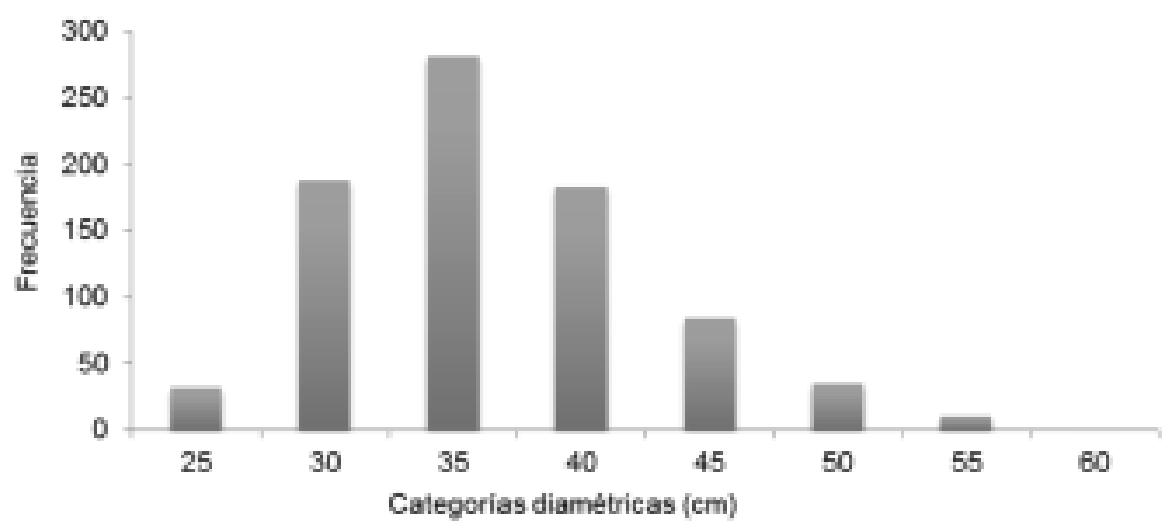

Figura 1. Frecuencia de categorías diamétricas de 815 trozas de Lonchocarpus castilloi Standl. 


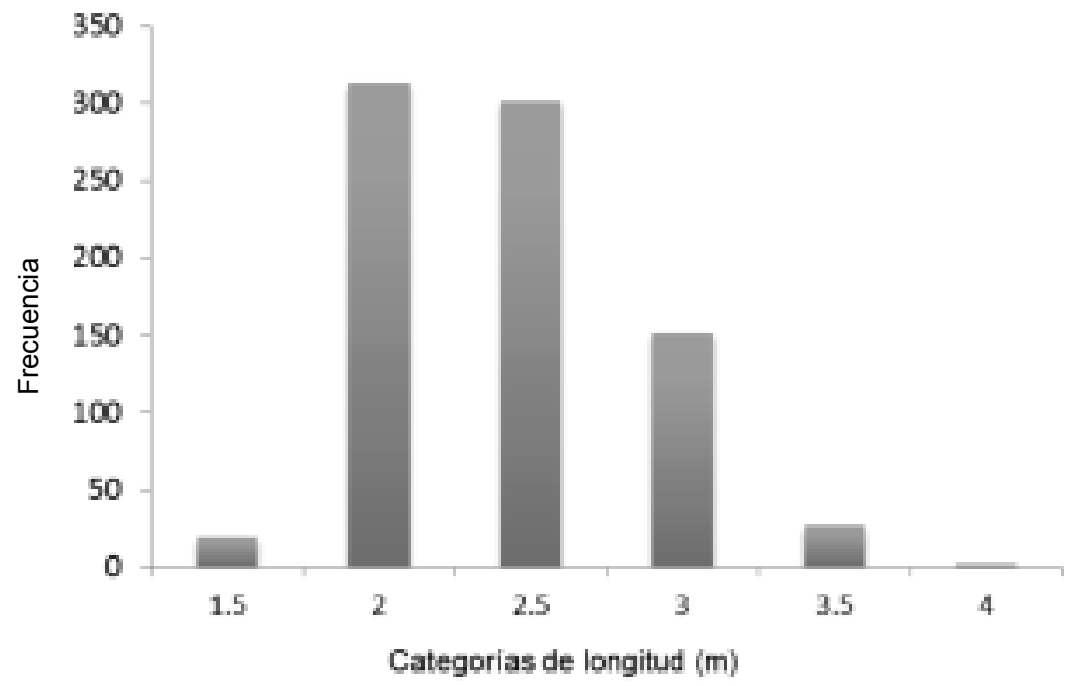

Figura 2. Frecuencia en categoría de longitudes de 815 trozas de Lonchocarpus castilloi Standl.

los fustes se dimensionan a diferentes longitudes para conseguir la mayor rectitud de la troza y eliminar presencia de defectos de sanidad como: pudrición, quemaduras, fuste hueco, entre otros, aunado a lo anterior madera aserrada de largas dimensiones tiene un mayor precio. Según la capacidad del aserradero y la especie, se procesan trozas de $1,10 \mathrm{~m}$ a $4,87 \mathrm{~m}$. Por las características de los fustes de machiche, para evitar que exista una mayor proporción de madera no calibrada, se ha determinado a través de la experiencia que $4,06 \mathrm{~m}$ es la longitud máxima de la troza que puede desplazar el carro en condiciones normales de trabajo.

Ochenta por ciento de las trozas aserradas presentaron diámetros de $30 \mathrm{~cm}$ a $40 \mathrm{~cm}$; y $94 \%$ presentaron longitudes de $2 \mathrm{~m}$ a $3 \mathrm{~m}$. Los resultados de otros estudios, como el de Herrera (2008), quien también trabajó con Lonchocarpus castiIloi, con diámetros de $40 \mathrm{~cm}$ a $70 \mathrm{~cm}$ y el de Valera y Salvador (2009), quienes trabajaron con Iryanthera laevis Margrak y Simarouba amara Aubl, con diámetros de $28,1 \mathrm{~cm}$ a $59,4 \mathrm{~cm}$; son comparables con los valores dasométricos promedio de las 815 trozas en el presente trabajo. El diámetro promedio fue de 36,55 centímetros y la longitud promedio de 2,41 metros (Tabla 2).

El volumen sin corteza se determinó aplicando una disminución de $10 \%$ al volumen con corteza, de acuerdo con Reuter et al. (1998), quienes mencionan que cerca de $10 \%$ del volumen de maderas duras es representado por la corteza.

Procesamiento primario de la madera. La trocería se aserró empleando el sistema de corte mixto, que es de uso tradicional. El total de las trozas del estudio, sumaron un volumen de 194,62 $\mathrm{m}^{3}$ rollo fuste sin corteza ( $r f s c)$, que al aserrarse se obtuvo un volumen 34252 pies tabla (pt). Este volumen, al ser trans- 
Tabla 2. Número, volumen y dimensiones de las 815 trozas empleadas de Lonchocarpus castilloi Standl.

\begin{tabular}{lcccccccc}
\hline $\begin{array}{c}\text { Cantidad } \\
\text { de trozas }\end{array}$ & $\begin{array}{c}\text { Volumen } \\
m^{3} \text { rollo c. c }\end{array}$ & $\begin{array}{c}\text { Volumen } \\
m^{3} \text { rollo s. c }\end{array}$ & \multicolumn{2}{c}{$\begin{array}{c}\text { Diámetro } \\
\text { de trozas }(\mathrm{cm}) \\
\text { Mínimo Medio }\end{array}$} & \multicolumn{2}{c}{$\begin{array}{c}\text { Longitud } \\
\text { de trozas }(\mathrm{m})\end{array}$} \\
\hline 815 & 216,2 & 194,6 & 23 & 36 & 57 & 1,5 & 2,4 & 4,0 \\
\end{tabular}

formado al Sistema Internacional de Unidades correspondió a $80,78 \mathrm{~m}^{3}$ de madera aserrada, resultantes de la cubicación de 19552 piezas de madera aserrada de largas y cortas dimensiones. Lo anterior indica que en este aserradero se requieren $2,45 \mathrm{~m}^{3} \mathrm{rfsc}$ de Lonchocarpus castilloi Standl para producir un metro cúbico de madera aserrada. En la tabla 3 se presentan los valores extremos y medios de madera en rollo y productos aserrados.

Durante el tiempo de asierre de las 815 trozas (Fig. 3), se presentó un coeficiente de variación de $32,23 \%$. Mientras que el coeficiente de variación en la producción diaria de los productos aserrados obtenidos fue de 35,65\% (Fig. 4). Algunos autores mencionan que la variación en el asierre y en la producción refleja problemas operativos en la producción del aserradero. Fuentes-López et al. (2008) sugieren que altos coeficientes de variación y bajos volúmenes de producción de madera aserrada, se originan principalmente por la falta de una planea- ción e infraestructura tecnológica adecuada. Esta situación es común en la mayoría de los aserraderos pequeños y medianos de Quintana Roo.

En la tabla 4 se muestra el volumen de la madera aserrada y sus proporciones de acuerdo con sus dimensiones y calidad, mientras que en la figura 5 se representa la relación del volumen de tablas obtenido en la producción.

Las tablas con contenido de albura y de duramen, que representan $77 \%$ de la producción, tienen un precio de venta favorable. Mientras que las tablas tipo rechazo ( $23 \%$ de la producción), representan una carga para los costos de producción, debido a que este último tipo de madera es de calidad inferior $y$ presenta poca demanda en el mercado, tanto local como externo. Los datos de la tabla 4 y de la figura 5 , indican las posibilidades económicas y la rentabilidad potencial del asierre de madera de Lonchocarpus castilloi Standl, bajo las condiciones actuales del aserradero.

Tabla 3. Volúmenes mínimo, medio y máximo, de madera en trozo y aserrada de 815 trozas de Lonchocarpus castilloi Standl.

\begin{tabular}{cccccc}
\hline \multicolumn{2}{c}{ Volumen de madera en rollo $\left(m^{3}\right)$} & \multicolumn{3}{c}{ Volumen de productos aserrados $\left(m^{3}\right)$} \\
Mínimo & Medio & Máximo & Mínimo & Medio & Máximo \\
\hline \multirow{2}{*}{3,802} & 10,2 & 13,5 & 1,5 & 4,2 & 6,1 \\
\hline
\end{tabular}




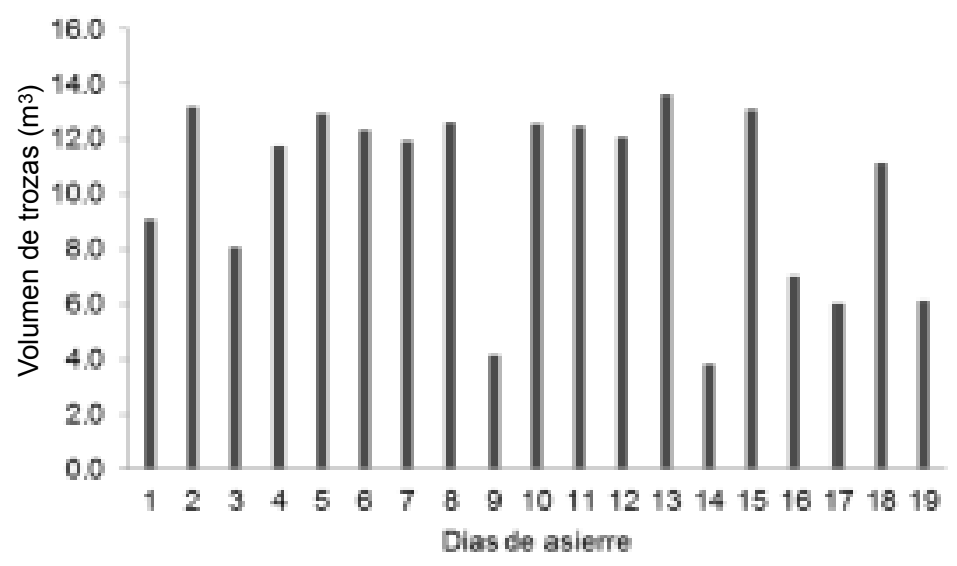

Figura 3. Relación del asierre de Lonchocarpus castilloi Standl, durante los días de trabajo.

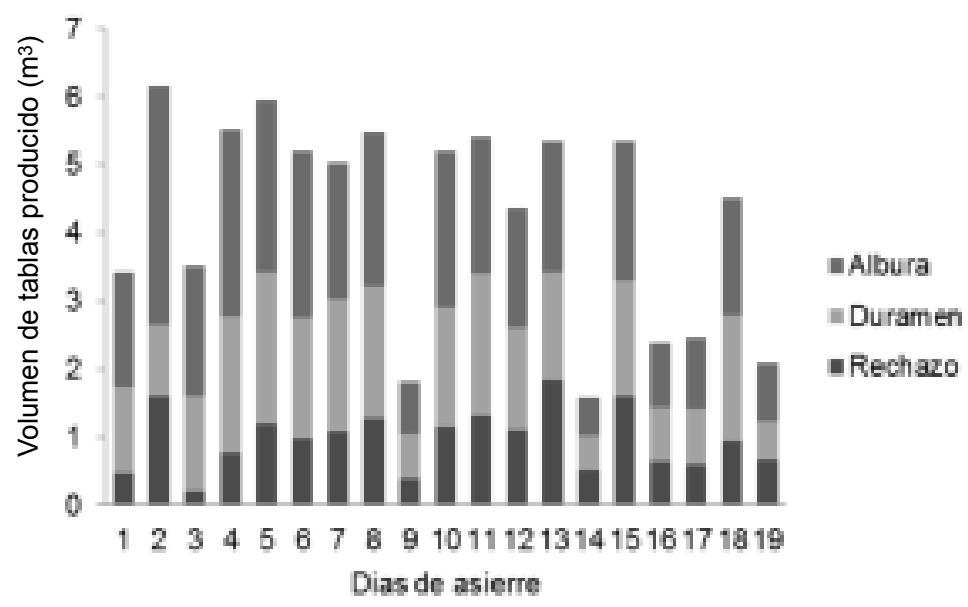

Figura 4. Relación de la producción de madera aserrada de Lonchocarpus castilloi Standl, por calidades durante los días de trabajo. 
Tabla 4. Volumen de madera aserrada de Lonchocarpus castilloi Standl, por dimensiones y tipos de productos.

\begin{tabular}{lccccc}
\hline Dimensiones & \multicolumn{4}{c}{ Volumen por tipos de productos aserrados (m3) } & Proporción (\%) \\
\hline & Albura & Duramen & Rechazo & Total & \\
\hline Cortas dimensiones & 16,7 & 16,41 & 4,6 & 34,6 & 42,8 \\
Largas dimensiones & 18,3 & 14,1 & 13,8 & 46,2 & 57,2 \\
Total & 35,0 & 27,5 & 18,3 & 80,8 & 100 \\
Proporción (\%) & $43 \%$ & $34 \%$ & $23 \%$ & $100 \%$ & \\
\hline
\end{tabular}

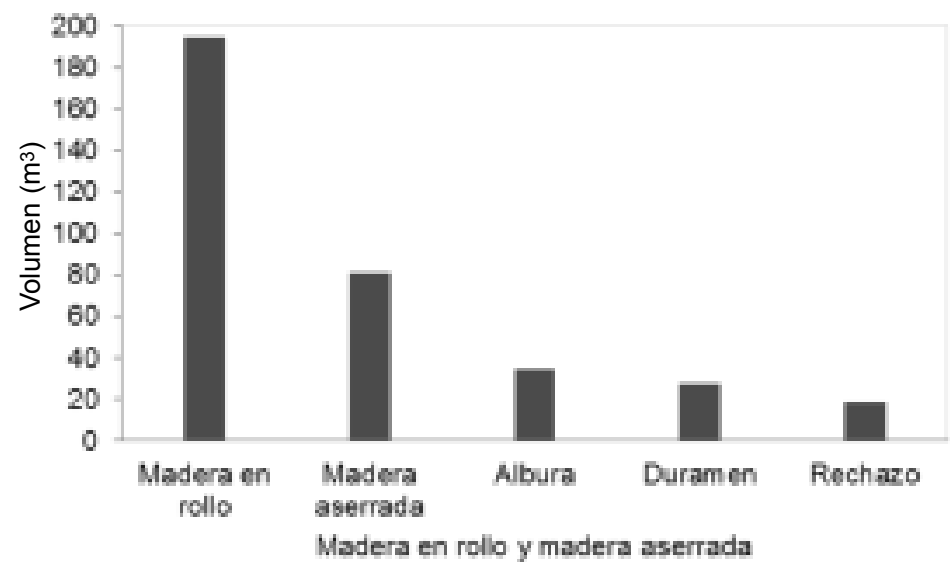

Figura 5. Relación del volumen de la madera aserrada de Lonchocarpus castilloi Standl, con respecto al volumen de la madera en rollo.

Al obtenerse una mayor proporción de madera de albura, es deseable diversificar sus productos. Adicional a la elaboración de duela y parquet, entre los usos recomendados por Bárcenas (1995) para Lonchocarpus castilloi Standl, se encuentran la elaboración de pilotes, productos tor-neados y para usos estructurales en exteriores.

Para la administración del aserradero, conocer el número de piezas obtenidas, de acuerdo con su calidad (Tabla 5 ), permite proyecciones concretas de los ingresos potenciales.
Aunque las proporciones y el número de tablas producidas se consideran aceptables debido a las condiciones prevalecientes en el aserrado de Lonchocarpus castilloi Standl; como lo recomiendan diversos autores, es deseable para maderas duras valuar el rendimiento de diversos sistemas de aserrado, con el fin de optimizar la eficiencia del aserradero y de la materia prima (Brown y Bethel, 1990; Zavala, 1994; Zavala, 2003 y Nájera et al., 2006).

Coeficiente de aserrío (CA). El coeficiente de aserrío real de las 815 trozas de 
Tabla 5. Productos obtenidos del aserrío de Lonchocarpus castilloi Standl, por tipo de calidad.

\begin{tabular}{lcccccc}
\hline Productos obtenidos del aserrío & \multicolumn{3}{c}{ Albura } & \multicolumn{5}{c}{ Tipo de calidad de los productos del aserrío } \\
& Largas & Cortas & Largas & Cortas & Largas & Cortas \\
& 2602 & 1930 & 2593 & 5123 & 1930 & 2018 \\
Tablas generadas & 18,29 & 16,68 & 14,11 & 13,36 & 13,77 & 4,55 \\
\hline
\end{tabular}

Tabla 6. Coeficiente de Aserrío real de Lonchocarpus castilloi Standl, por dimensiones y tipos de productos aserrados.

\begin{tabular}{|c|c|c|c|c|}
\hline \multirow[t]{2}{*}{ Dimensiones } & \multicolumn{4}{|c|}{ Coeficiente de Asierre (\%) } \\
\hline & Albura & Duramen & Rechazo & Total \\
\hline Cortas dimensiones & 8,48 & 6,99 & 2,52 & 18,00 \\
\hline Largas dimensiones & 9,33 & 7,11 & 6,89 & 23,34 \\
\hline Total & 17,82 & 14,11 & 9,41 & 41,34 \\
\hline
\end{tabular}

Lonchocarpus castilloi Standl (Tabla 6), se obtuvo para las dos longitudes y las tres calidades establecidas. Con base en el rendimiento obtenido de $41,34 \%$ sin corteza, se determinó que en el aserradero se obtienen 175 pies tabla por cada metro cúbico en rollo procesado de machiche.

El volumen promedio y el rendimiento permiten a la administración del aserradero medir la eficiencia del proceso productivo y realizar los ajustes necesarios para incrementarlo. Este coeficiente de aserrío $(41,34 \%)$ resultó superior que el coeficiente de $35,22 \%$ calculado para la misma especie en un estudio realizado por Acosta (1999). Tal diferencia puede deberse principalmente a la falta de especialización de los operarios clave; al considerar que fue empleado el mismo equipo de aserrado y se trabajó con un diámetro medio de $43 \mathrm{~cm}$. Al respecto, Nájera et al. (2011) mencionan que entre los principales factores que afectan el rendimiento de madera aserrada, destacan el diámetro, clase, calidad y forma de las trozas, los tiempos empleados en el proceso, el patrón de corte, el tipo de sierra, las dimensiones de los productos a obtener, la especialización de los operarios y las condiciones del equipo de aserrío. Comparado con el estudio de Herrera (2008), que obtuvo un rendimiento de $41,61 \%$ para machiche, el coeficiente obtenido en este trabajo resulta similar.

Si se toma como base de comparación el intervalo de coeficientes de rendimiento de $35 \%$ a $40 \%$ encontrado por Reuter et al. (1998), determinado para maderas duras tropicales, el coeficiente obtenido en este trabajo resulta ligera- 
mente superior. Aunque este coeficiente resultó inferior, para el rendimiento de $45 \%$ a $55 \%$, determinado para especies frondosas por Rocha (2002). También es inferior a $58 \%$, establecido para especies latifoliadas por El Instituto Nacional de Bosques, citado por Herrera (2008).

Los residuos generados por el aserrado de Lonchocarpus castilloi Standl, sumaron $58,66 \%$, los cuales resultan altos al comparar con los valores de $40,17 \% ; 46,10 \%$ y $37,37 \%$ calculados por Biasi y Rocha, (2006) en el aserrío de Erisma uncinatum, Mezilaurus itauba y Qualea albiflora, respectivamente. Como indican estos mismos autores, tal vez el mayor problema que enfrenta la industria de la madera aserrada es el bajo aprovechamiento de materia prima, que acarrea un aumento en el costo del producto final y una gran generación de residuos. Por su parte, Velázquez et al. (2006) plantean que los altos niveles de residuos en un aserradero revelan falta de tecnologías apropiadas y la necesidad de pensar en alternativas que puedan suplir el déficit de ganancias de la industria por concepto de venta de madera aserrada. Si bien en Quintana Roo se han dado algunos avances importantes en la industria forestal ejidal, se requiere mejorar el proceso de aserrado de especies latifoliadas; esto es particularmente necesario debido a que la empresa forestal ejidal, desde 1994 está comprometida con la certificación forestal y los principios del Forest Stewardship Council, por medio de la evaluación del programa Smartwood de Rain Forest Alliance.

El nivel de aprovechamiento de la materia prima en general en un aserradero depende en grado considerable de los métodos utilizados para la elaboración de la madera aserrada (Álvarez-Lazo et al-, 2004; Murara et al., 2005). Un sistema de corte que genera mejores rendimientos en una especie, puede no ser el mejor sistema para otra especie. Lo anterior fue encontrado por Valera y Salvador (2009), quienes analizaron dos tipos de corte en dos especies. Para la especie Iryanthera laevi Margrak, registraron un rendimiento de $46,9 \%$, empleando el sistema de corte denominado al cuartón de brusellas, contra $66,8 \%$ obtenido empleando el sistema de corte tradicional. La segunda especie Simarouba amara Aubl registró un coeficiente de $44,9 \%$ con el sistema de aserrío al cuartón de brusellas, mientras que se obtuvo un rendimiento de $40,9 \%$ al emplear el sistema de aserrío tradicional.

De acuerdo con el análisis de correlación entre el volumen de madera en rollo y el volumen de madera aserrada por tipos de productos aserrados, se observa una favorable asociación entre éstas (Tabla 7). El grado de asociación mayor corresponde al volumen de madera en rollo con el volumen total de madera aserrada; la menor correlación es para su relación con el volumen de rechazo.

Una tendencia similar se observó en el análisis de correlación del volumen de madera en rollo y el número de piezas de tablas producidas por tipos de calidad (Tabla 8). Al igual que en la tabla 7 , la mayor dependencia fue para la madera en rollo con el número total de tablas aserradas y la menor asociación fue con las tablas de rechazo.

Se han realizado diversos estudios para determinar los factores que influyen en la tendencia del rendimiento en un lote de trozas. Autores como Zavala y Hernández (2000), Velázquez et al. (2006) y Nájera-Luna et al. (2011), encontraron que el rendimiento en madera aserrada decrece con el incremento en longitud. Al respecto Steele, (1984), señala que esta tendencia es debida al efecto de la forma de la troza, puesto que 
Tabla 7. Correlación del volumen de madera en rollo entre el volumen de madera aserrada de Lonchocarpus castilloi Standl.

$\begin{array}{ll}\text { Volumen de madera en rollo-volumen de tablas calidad duramen } & 0,84 \\ \text { Volumen de madera en rollo-volumen de tablas calidad albura } & 0,85 \\ \text { Volumen de madera en rollo-volumen de tablas calidad rechazo } & 0,82 \\ \text { Volumen de madera en rollo-volumen total de tablas } & 0,97\end{array}$

Tabla 8. Correlación del volumen de madera en rollo con el número de piezas de tablas producidas de Lonchocarpus castilloi Standl.

a mayor largo es probable que la troza se encorve y pierda rectitud ocasionando problemas para encontrar la adecuada geometría del aserrío.

Al analizar el rendimiento por categoría diamétrica, Zavala y Hernández (2000) y Nájera-Luna et al. (2011), no encontraron una tendencia de aumento con el incremento en diámetro de las trozas de coníferas en el rango de categorías diamétricas de $30 \mathrm{~cm}$ a $65 \mathrm{~cm}$. Murara et al. (2005) encontraron que el aserrío convencional no mostró tendencia de incremento con el aumento en diámetro de las trozas, mientras que con el aserrío optimizado el rendimiento fue mayor en todas las clases con el aumento en diámetro de las trozas. Para Nájera-Luna et al. (2011), el largo de las trozas influye en el rendimiento de madera aserrada, puesto que a medida que aumenta su longitud, el rendimiento disminuye pero se aumenta la productividad. Por su parte, Valera y Salvador (2009) encontraron una relación positiva altamente significativa entre el diámetro promedio de las trozas y el número de piezas de madera aserrada, por tanto, a menor número de cortes se obtiene un mayor rendimiento.

Entre el conjunto de recomendaciones emitidas por diversos autores, para contribuir a elevar la eficiencia de conversión en los aserraderos, se encuentran: acondicionar maquinaria y equipo para el aserrado de los diversos grupos de especies (García y Moya, 1998), especialmente porque a mayor calidad del aserrado se incrementa el volumen de madera aserrada; capacitar a los operarios clave en el asierre sobre normas de clasificación y control de calidad (Guillén y Chávez, 1996). Emplear programas de control de dimensiones en aserraderos y utilizar equipo con reducción de residuos (Steele et al., 1991; Álvarez-Lazo et al., 2004). Implantar un sistema de clasificación y selección de trozas, identificando clases diamétricas en los aserraderos (Murara et 
al., 2005). Investigar el contenido de humedad de trozas propicio (Quirós et al., (2005), para que se requiera un menor afilado de las sierras (García y Moya, 1998). Utilizar patrones de corte para cada clase de diámetro (García y Moya, 1998; Murara et al., 2005), según el tipo de producto a obtener (Aguilera et al., 2005; Biasi y Rocha, 2006 y García et al., 2009).

Determinación de tiempos. De acuerdo con los datos obtenidos del proceso de aserrado en la sierra principal, el tiempo total observado fue de 141,27 horas. Para Guillén y Chávez (1996), el tiempo trabajado productivo en la sierra principal, es considerado como el mejor indicador de la efectividad del aserradero. En el presente estudio, $68 \%$ del tiempo trabajado es productivo $(94,37$ horas), porcentaje que resulta similar al tiempo trabajado productivo determinado por Guillén y Chávez (1996) para el aserrado de Swietenia macrophylla (caoba) que es madera clasificada como blanda, mientras que Lonchocarpus castilloi Standl está clasificada por Bárcenas (1995) como madera dura y de difícil trabajabilidad en el aserrío. En la figura 6 se muestra la proporción de tiempos obtenidos con respecto al tiempo total observado.

El porcentaje del tiempo trabajado del presente estudio es $3,5 \%$ menor que el tiempo productivo de $71,42 \%$, encontrado por León (2001) para el aserrado de pinos. Por lo anterior, al considerar que la sierra de corte requería afilado constante al emplear madera de aserrado difícil, y por la falta de un tratamiento previo que facilite el aserrado de maderas duras, el resultado de esta clase de tiempo obtenido puede considerarse aceptable, aunque resulta inferior en $28,28 \%$ con el tiempo productivo de $96,28 \%$ para el asierre de pinos, estimado por Ramírez (1995) en Tepehuanes, Durango.

El tiempo trabajado no productivo en la sierra principal fue de 27,09 horas (19\%), el cual se distribuyó como se muestra en la figura 7. El afilado y el cambio de sierra emplearon $56 \%$ del tiempo trabajado no productivo, esto debido, principalmente, a la falta de una mayor cantidad de cintas de repuesto para el corte de maderas duras.

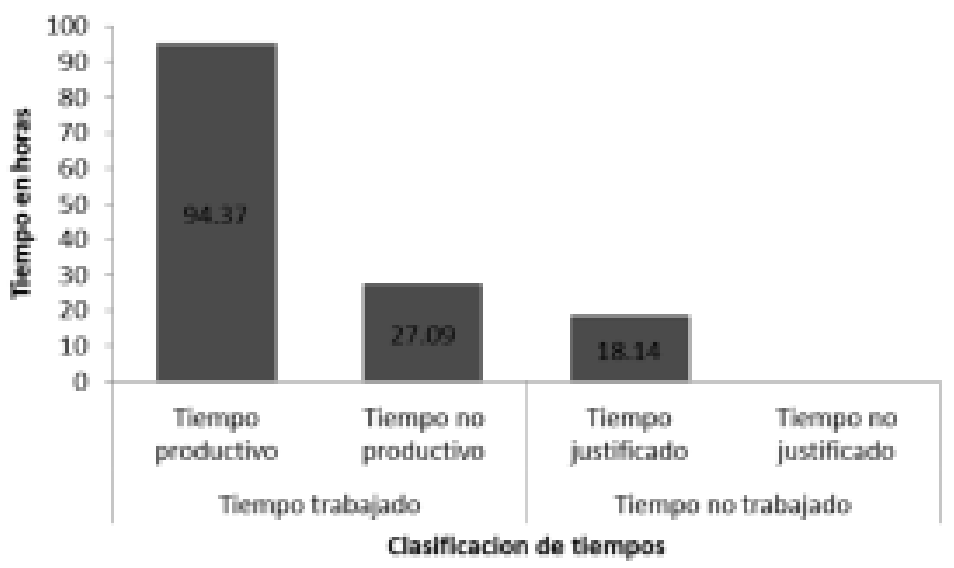

Figura 6. Distribución de tiempos en la producción de madera aserrada de Lonchocarpus castilloi Standl. 


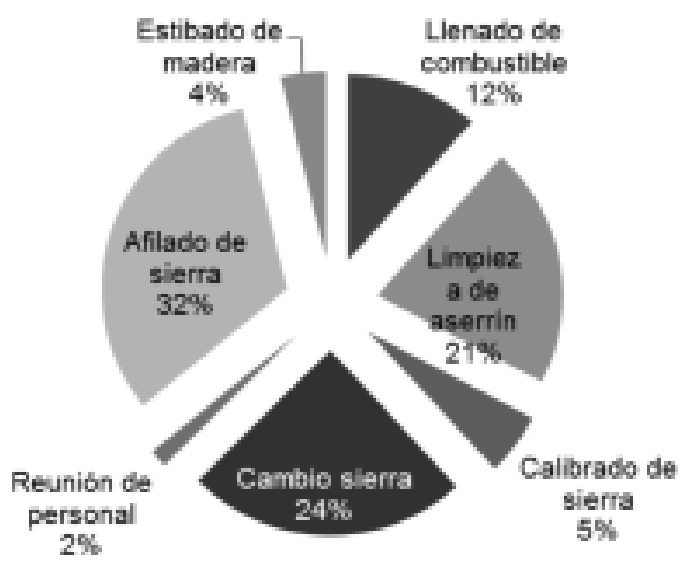

Figura 7. Distribución del tiempo trabajado no productivo en la sierra principal, durante el asierre de Lonchocarpus castilloi Standl.

Esta clase de tiempo obtenido puede considerarse elevado comparado con $3,71 \%$ encontrado por Ramírez (1995) para el aserrío de pinos y con $13,63 \%$; encontrado por Guillén y Chávez (1996) para el aserrío de caoba, pero es similar al 19,1\%, determinado por León (2001), quien realizó un estudio para coníferas en sierra sur del estado de Oaxaca.

El tiempo no trabajado justificado (Fig. 8), en la sierra principal, sumó 18,14 horas (13\%). Este tiempo se debió principalmente a la falta de un plan de operación anual del aserradero, y principalmente a la falta de capital para invertir en el mantenimiento del equipo de asierre. En Bolivia, Guillén y Chávez (1996) determinaron un $14,50 \%$ del tiempo no trabajado justificado para el asierre de caoba. Por su parte, León (2001) estimó 9,19\% del tiempo no trabajado justificado en el aserrado de coníferas.

En el aserradero ejidal Tres Garantías operan un total de 21 trabajadores, la producción de madera aserrada es función de 6 trabajadores; quienes en el aserrado de las 815 trozas produjeron un volumen de $80,78 \mathrm{~m}^{3}$. La variabilidad del asierre se reflejó en el tiempo trabajado y en el tiempo no trabajado. En el día menos productivo se trabajó durante dos horas, mientras que en el día más productivo se trabajó por 6,95 horas. En promedio se trabajó 4,86 horas por día.

Determinación de la productividad. Por hora laborable en el aserradero en promedio se procesaron 5,76 trozas, mientras que la producción del volumen de madera aserrada por jornada por hora se ubicó en 0,027 $\mathrm{m}^{3}$. La productividad, que corresponde al volumen en metros cúbicos de madera aserrados por hora, indica que en promedio se procesaron $0,571 \mathrm{~m}^{3} / \mathrm{h}$. Este resultado es inferior, con respecto a otros estudios realizados, como la productividad de $6,62 \mathrm{~m}^{3} / \mathrm{h}$ encontrada por Ramírez (1995) en Durango; la de $3,49 \mathrm{~m}^{3} / \mathrm{h}$ calculada por García et al. (2001), quienes trabajaron en cuatro aserraderos en el estado de Jalisco; la de $2,17 \mathrm{~m}^{3} / \mathrm{h}$ calculada por Estévez et al. (2010), empleando un aserradero portátil; y el valor más 


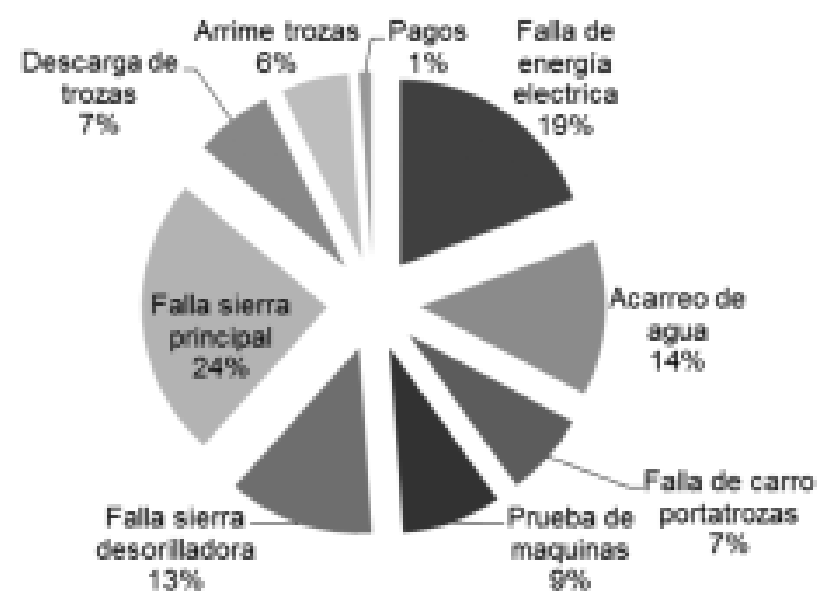

Figura 8. Distribución del tiempo no trabajado justificado en la sierra principal, durante el asierre de Lonchocarpus castilloi Standl.

elevado, obtenido por Nájera et al. (2011) de $7,57 \mathrm{~m}^{3 / \mathrm{h}}$, para la región de El Salto, Durango, en el aserrado de encinos y pinos. En sentido estricto, una comparación de productividades del aserrado de coníferas con la productividad obtenida en este estudio, puede no ser válida, por tanto es empleada únicamente como referencia al ser la única información disponible. Adicionalmente debe considerarse que la productividad de $0,571 \mathrm{~m}^{3} / \mathrm{h}$, está en función de los objetivos de producción del aserradero; en este estudio, los objetivos del aserrado de machiche consistieron en obtener el mayor volumen de madera de largas dimensiones con contenido exclusivo de duramen.

Autores como Murara et al. (2005) y Nájera-Luna et al. (2011) encontraron que la productividad incrementa a medida que aumenta el diámetro, la longitud de las trozas y la velocidad de alimentación. En este caso, la productividad determinada refleja una baja eficiencia por varios factores que influyen en diversas intensidades, tales como el deterioro de los fustes en patio por un año, la falta de hidratación de las trozas previo al aserrado, limitaciones tecnológicas para el asierre de maderas duras y la falta de capitalización para el mantenimiento previo al periodo de asierre anual. Los valores mencionados indican que el aserradero está enfrentando altos costos de producción que afectan directamente las utilidades.

De acuerdo con Jiménez, citado por Ramírez (1995), toda empresa que desee mejorar su productividad y rentabilidad, tendría que considerar la reducción de tiempos no productivos y ociosos a $5 \%$ del tiempo total. Mientras que, Adams y Helvoigt (2008) mencionan que la mayor parte del crecimiento de la productividad de la industria del aserrío tiene su origen en cambios técnicos para el asierre. Por tanto es deseable, con el fin de incrementar la productividad, actualizar el programa de desarrollo forestal industrial, 
adecuar el equipo para el asierre de maderas tropicales, programar capacitación, desarrollar investigación y monitorear la productividad.

\section{CONCLUSIONES}

Las 815 trozas del estudio sumaron un volumen de 194,6 $\mathrm{m}^{3}$ rollo fuste sin corteza (rfsc), que al ser aserrados, proporcionaron un volumen $80,8 \mathrm{~m}^{3}$ de madera aserrada. Se requieren $2,45 \mathrm{~m}^{3}$ rfsc de Lonchocarpus castilloi Standl para producir un metro cúbico de madera aserrada.

El coeficiente de aserrío real total fue de $41,3 \%$, del cual $17,8 \%$ correspondió a madera de albura, $14,1 \%$ a madera de duramen y $9,41 \%$ a madera de rechazo.

El tiempo trabajado productivo fue de $68 \%$. El tiempo trabajado no productivo fue de $19 \%$, el tiempo no trabajado justificado se ubicó en 13\%. La productividad en el aserrado de Lonchocarpus castilloi Standl se ubicó en $0,57 \mathrm{~m}^{3} / \mathrm{h}$.

Con el propósito de incrementar la productividad en el proceso de aserrado, se recomienda: mejorar las prácticas de mantenimiento de la maquinaria y equipo, mejorar las herramientas de corte, mejorar la capacitación de afiladores y aserradores, sobre todo en el uso de plantillas de aserrado y ajuste de sierras a requerimiento, especialmente por la utilización de la misma maquinaria en el aserrado de maderas blandas y duras tropicales, clasificar las trozas por calidades, en patio; como tratamiento previo al aserrío hidratar y eliminar incrustaciones en las trozas de maderas duras que así lo requieran y considerar la estandarización de longitudes en la troza. Es deseable continuar con estudios de rendimiento de aserrado en maderas tropicales, considerar elementos como, calidad de trocería y clasificación en categorías de diámetro y longitud, velocidad de alimentación, inclinación y ancho del ángulo de ataque, variación del corte, tipo y características de la sierra de corte, calidad de la madera aserrada, evaluar otros sistemas de corte. También es deseable realizar un estudio de tiempos a detalle, donde se evalúe la eficiencia y productividad de la maquinaria y equipo y la del personal.

\section{RECONOCIMIENTOS}

Se reconoce a la Sociedad de Productores forestales Ejidales del Sur de Quintana Roo (SPFEQRoo), al administrador del aserradero ejidal y a los ejidatarios de Tres Garantías, por el interés en la realización de este estudio.

\section{REFERENCIAS}

Acosta R., A. 1999. Coeficientes de aserrío para las especies Machiche (Lonchocarpus castilloi Standl) y Chechen (Metopium brownei (Jacq) Urban), en los aserraderos ejidales de Noh-bec y Tres Garantías, Quintana Roo, México. Memoria de residencia profesional. Instituto Tecnológico Agropecuario núm. 16, Juan Sarabia, Quintana Roo. México. $51 \mathrm{p}$.

Adams, D.M y T. Helvoigth L. 2008. Data envelopment analysis of technical efficiency and productivity growth in the US Pacific Northwest sawmill industry. Canadian Journal of Forestry Research 38(10):22532565.

Aguilera, A., L. Inzuza, R. Alzamora y L. Tapia. 2005. Evaluación del costo de producción para faenas de aserrío portátil. Bosque 26(2):107-114. 
Álvarez-Lazo, D., E. Andrade-Fernando, G. Quintín-Cuador y A. DomínguezGoizueta. 2004. Importancia del control de las dimensiones de la madera aserrada. Revista Chapingo, serie Ciencias Forestales y del Ambiente 10(2):105-110.

Ayerde L., D. 1993. Metodología para la determinación de coeficientes de aserrío. SARH-INIFAP-CIR. Folleto técnico núm. 1. Chilpancingo, Gro. $19 \mathrm{p}$.

Bárcenas P., GM. 1995. Caracterización tecnológica de veinte especies maderables de la Selva Lacandona. Madera y Bosques 1(1):9-38.

Bennett, B.F. 1972. Marginal logs and profitability of low investment sawmills. Journal of Forestry (2):26-27.

Biasi C., P. y M.P. Rocha. 2006. Rendimento em madeira serrada e cuantificacao de residuos para tres especies tropicais. Floresta 37(1):95-108.

Brown C., N. y J.S. Bethel. 1990. La industria maderera. Séptima reimpresión, Editorial Limusa Noriega. México D.F. $397 \mathrm{p}$.

Chávez, A. y A. Guillén. 1997. Estudio de rendimiento, tiempos y movimientos en el aserrío. Manual práctico. Documento técnico 62.Proyecto BOLFOR. Santa Cruz Bolivia. 29 p.

Estévez M., W.L., G.I. Bolzon M, M.G. Lomelí y D.C. Bautista. 2010. Estudio de la productividad de corte en madera de Pinus elliottii utilizando un prototipo de aserradero móvil. Maderas, Ciencia y Tecnología 12(1):43-52.

Flores-Velázquez, R., E. Serrano-Gálvez, V.H. Palacio-Muñoz y G. Chapela. 2007. Análisis de la industria de la madera aserrada en México. Madera y Bosques 13(1):47-59.

Forster, R., H. Albrecht, M. Belisle, A. CabaIlero, H. Galletti, O. Lacayo, S. Ortiz y D. Robinson. 2002. Comunidades forestales y el mercadeo de maderas tropicales poco comerciales de Mesoamérica. Universidad de Quintana Roo, USAID, USDA. México. 156 p.

Fuentes-López, M.E., J.A. García Salazar y J. Hernández Martínez. 2006. Factores que afectan el mercado de madera aserrada de pino en México. Madera y Bosques 12(2):17-28.

García R., J.D., L. Morales y S. Valencia. 2001. Coeficientes de aserrío para cuatro aserraderos banda del Sur de Jalisco. Foresta-AN. Nota Técnica núm. 5. UAAAN, Saltillo, Coah. 12 p.

García D., J.M., O. Izquierdo, P.P. Henry y A. Domínguez G. 2009. Algunos aspectos relacionados con la calidad de la madera aserrada de Pinus caribaea Morelet var. Caribaea. Ra Ximhai 5(3):259-270.

García S., R. y R. Moya. 1998. El aserrío de Dialium guianense (Aubl) Sandwit en Costa Rica. Madera y Bosques 4(1):41-51.

Guillén, A. y J. Chávez. 1996. Estudio de tiempos y rendimientos en aserrío de Mara en un aserradero de cinta en Santa Cruz. BOLFOR. Bolivia. Bol. núm. 6:9-10.

Gutiérrez P., H. y S.R. De la Vara. 2008. Análisis y diseño de experimentos. $2 a$ ed. McGraw-Hill Interamericana. México. 545 p.

Herrera T., W.D. 2008. Monitoreo de actividades de aprovechamiento forestal sostenible, realizado en la unidad de 
manejo Uaxactún reserva de la biosfera Maya, Petén Guatemala. Tesis de licenciatura. Universidad de San Carlos de Guatemala. Guatemala. $133 \mathrm{p}$.

Juacida R., P. y E. Saldivar. 2000. Aserrío y secado de tres cultivares de Populus $x$ euramericana (Dode) Guinier creciendo en Valdivia, Chile. Bosque 21(1):65-71.

León M., J.S. 2001. Análisis del coeficiente de asierre del aserradero de San Pedro el alto, Zimatlán, Oaxaca. Tesis de maestría. Instituto Tecnológico Agropecuario núm. 23. Ex-hacienda de Nazareno, Xoxocotlán, Oaxaca, México. $136 \mathrm{p}$.

Martínez-Pastur, G., J.M. Cellini, M.V. Lencinas, B. Díaz, P.L. Peri y R.F. Vukasovic. 2002. Funciones de rendimiento volumétrico en pie y en aserradero para la Lenga (Nothofagus pumilio). Ciencias Forestales 15(12):32-45.

Meneses, M. y S. Guzmán. 2000. Productividad y eficiencia de la producción forestal basada en plantaciones de Pino radiata. Bosque 21(2):3-11.

Moya R., R. y R. Córdoba. 1995. Evaluación del aserrío y trabajabilidad de madera de Pochote (Bombacopsis quinatum) de ocho años de edad. Revista Forestal Centroamericana 4(12):19-30.

Murara M., I., M.P. da Rocha y R. Timofeikzyc. 2005. Rendimento em madeira serrada de Pinus taeda para duas metodologías de desdobro. Floresta 35(3):473-483.

Nájera-Luna, J.A., I. Rodríguez R., J. Méndez G., J.J. Graciano L., F. Rosas y F.J. Hernández. 2006. Evaluación de tres sistemas de asierre en Quercus sideroxyla Humb \& Bompl., de El Salto, Durango. Ra Ximhai 2(2):497-513.

Nájera-Luna, J.A., O.A Aguirre-Calderón, E.J Treviño-Garza, J. Jiménez-Pérez, E. Jurado-Ybarra, J.J. Corral-Rivas y B. Vargas-Larreta. 2011. Tiempos y rendimientos del aserrío en la región de El Salto, Durango, México. Revista Chapingo, serie ciencias forestales y del ambiente 17(2):199-213.

Quirós, R., O. Chinchilla y M. Gómez. 2005. Rendimiento en aserrío y procesamiento primario de madera proveniente de plantaciones forestales. Agronomía Costarricense 29(2):7-15.

Ramírez H., M. 1995. Tiempos y movimientos para un aserradero fijo con sierra banda en Tepehuanes Durango. Tesis de licenciatura. Universidad Autónoma Agraria Antonio Narro. División de Agronomía. Buenavista, Saltillo, Coahuila México. 57 p.

Reuter, M., C. Schulz y C. Marrufo. 1998. Manual Técnico Forestal. Información básica, métodos y procedimientos. Deutsche Gesellschaft fur Technische Zusammenarbeit $\mathrm{GmbH}$. Secretaría de Medio Ambiente Recursos Naturales y Pesca. Acuerdo México-Alemania. Hidroeléctrica infiernillo 157, Chetumal, Quintana Roo, México. 133 p.

Rocha M., P. 2002. Técnicas e planejamento em serrarias. Ed. rev. ampl. Curitiba: Fundaçăo de Pesquisas Florestais do Paraná.121 p

Romahn, C., H. Ramírez y J.L. Treviño. 1994. Dendrometría. División de Ciencias Forestales. Universidad Autónoma Chapingo. Chapingo, México. 353 p.

Rueda-Sánchez, A., A. Gallegos-Rodríguez, D. González-Eguiarte, J. de D. Benavides-Solorio, J.A Ruiz-Corral y E. 
López-Alcocer. 2007. Coeficiente de aserrío de madera en rollo de plantaciones forestales de especies tropicales en Jalisco. VIII Congreso Mexicano de Recursos Forestales. Michoacán. 13 p.

Steele, P. H. 1984. Factors determining lumber recovery in sawmilling. Gen. Tech. Rep.FPL- 39. Madison, WI: U.S. Department of Agriculture, Forest Service, Forest Products Laboratory. $8 \mathrm{p}$.

Steele, P.H., F.G. Wagner y K.E. Skog. 1991. Regional softwood sawmill processing variables as influencend by productive capacity. United States Department of Agriculture Forest Service.15 p.

Valera D., L.M. y M.I. Salvador C. 2009. Análisis de dos tipos de corte en el aserrío de la madera de dos especies forestales de la zona de Pucallpa. Revista Forestal UCAYALY 5(1):5-32.

Valerio A., F., L. Farinha W, R. Tavolca, C. Brandelero y H. Soares K. 2007. Cuan- tificação de resíduos e rendimento no desdobro de Araucaria angustifolia (BERTOL) O. KUNTZE. Floresta 37(3):387-398.

Velázquez D., D., K. Manzanares A, M.A. Guyat y E. Sánchez T. 2006. Rendimiento de materia prima en un aserradero de la provincia de Pinar del Rio Cuba. Revista Forestal Baracoa 25(1):97-104.

Zavala Z., D. 1994. Control de calidad en la industria de aserrío y su repercusión económica. INIFAP. Bol. Téc. núm. 115. Coyoacán, D.F. México. 48 p.

Zavala Z., D. 2003. Efecto del sistema de aserrío tradicional en las características de la madera de encinos. Madera y Bosques 9(2):29-39.

Zavala Z., D. y R. Hernández C. 2000. Análisis del rendimiento y utilidad del proceso de aserrío de trocería de pino. Madera y Bosques 6(2):41-55.

Manuscrito recibido el 19 de abril de 2012.

Aceptado el 21 de junio de 2012.

Este documento se debe citar como:

Acosta-Ramos, A. y J. Martínez-López. 2012. Productividad del aserrado de machiche (Lonchocarpus castilloi Standl), en Tres Garantías, Quintana Roo, México. Madera y Bosques 18(2):7-26. 